\title{
First report of tobacco vein banding mosaic virus infecting Solanum lyratum Thunb in China
}

\author{
Jian Zhang ${ }^{1,2,3} \cdot$ Yong-Yuan Cheng ${ }^{2,3} \cdot$ Lei Yang ${ }^{2,3} \cdot$ Xin Sun $^{2,3} \cdot$ Jing Yu ${ }^{2,3} \cdot$ Quan-You Lu ${ }^{1,2,3}$ (D)
}

Received: 28 August 2019 / Accepted: 1 October 2019 / Published online: 10 November 2019

(C) Società Italiana di Patologia Vegetale (S.I.Pa.V.) 2019

Keywords Solanum lyratum Thunb $\cdot$ Vein banding and mosaic symptoms $\cdot$ Tobacco vein banding mosaic virus

Solanum lyratum Thunb, a species of the genus Solanum in the family Solanaceae, is a common Chinese herbal medicine. Tobacco vein banding mosaic virus (TVBMV) is a member of the genus Potyvirus and was first discovered in Taiwan in 1964 (Habera et al. 1994). In July 2018, several S. lyratum plants growing naturally along a roadside exhibited virus-like symptoms including dark green vein banding and mosaic in Zhenjiang, Jiangsu province, China. The crude sap from the symptomatic leaves of $S$. lyratum was rubbed onto leaves of Nicotiana benthamiana plants, resulting in mild downward leaf curling and mild mosaic symptoms at 9 days post inoculation (dpi) and severe downward leaf curling, yellowing and plant stunting at $20 \mathrm{dpi}$, indicating mechanical transmission of a virus. Mock-inoculated $N$. benthamiana plants did not exhibit any symptoms at 9 or 20 dpi. Double-stranded (ds) RNA was extracted from the symptomatic leaves of $S$. lyratum (Morris and Dodds 1979), and used as a template for sequence-independent amplification (SIA) (Agindotan et al. 2010). Polymerase chain reaction (PCR) products were ligated into pUCm-T Vector (Sangon, Shanghai, China) and sequenced (SunYa, Shanghai, China). BLAST analysis showed that the nucleotide sequence (nt) of seven fragments were 92-

Electronic supplementary material The online version of this article (https://doi.org/10.1007/s42161-019-00419-y) contains supplementary material, which is available to authorized users.

Quan-You Lu sriluqy@163.com

1 Sericultural Research Institute, Jiangsu University of Science and Technology, Zhenjiang, Jiangsu 212018, People's Republic of China

2 College of Biotechnology, Jiangsu University of Science and Technology, Zhenjiang, Jiangsu 212018, People's Republic of China

3 Key Laboratory of Genetic Improvement of Silkworm and Mulberry, Ministry of Agriculture and Rural Affairs, Sericultural Research Institute, Chinese Academy of Agricultural Sciences, Zhenjiang, Jiangsu 212018, People's Republic of China
94\% identical to a TVBMV isolate from Shandong, China (JN630469). The presence of TVBMV was further confirmed in the symptomatic S. lyratum and $N$. benthamiana plants inoculated with crude sap by reverse transcription (RT) PCR using virus-specific primers (forward, 5'-GGATATTG GTGAGCAGATGC-3'; and reverse, 5'-CGTTTTCG ATGCACCACACCATC-3') designed on the sequences obtained by SIA. The expected 1109 nt product was not amplified from the asymptomatic or the mock-inoculated $N$. benthamiana plants. Subsequently, the complete genome of this isolate (tentatively named TVBMV-lyr) was determined by sequencing RT-PCR products obtained using degenerate TVBMV primers designed from the results of multiple sequence alignments of TVBMV isolates along with TMVlyr-specific primers designed from sequences obtained by SIA. The complete genomic sequence of TVBMV-lyr was deposited in GenBank as accession number MH898883. It is $9570 \mathrm{nt}$ in length and shares the highest nucleotide identity (94.32\%) with TVBMV-SDZC1 (HQ396793). To our knowledge, this is the first report of TVBMV infection in S. lyratum.

Acknowledgements This work was partially granted by the Natural Science Foundation of the Jiangsu Higher Education Institutions of China (No. 18KJA210001).

\section{References}

Habera LF, Berger PH, Reddick BB (1994) Molecular evidence from 3'terminus sequence-analysis that tobacco vein-banding mosaic virus is a distinct member of the Potyvirus group. Arch Virol 138:27-38 Agindotan BO, Ahonsi MO, Domier LL, Gray ME, Bradley CA (2010) Application of sequence-independent amplification (SIA) for the identification of RNA viruses in bioenergy crops. J Virol Methods 169:119-128

Morris TJ, Dodds JA (1979) Isolation and analysis of double stranded RNA from virus-infected plant and fungal tissue. Phytopathology 69(8):854-858

Publisher's note Springer Nature remains neutral with regard to jurisdictional claims in published maps and institutional affiliations. 